Goal-Dependent Learning in the Colour-Word Contingency Learning Paradigm: Adding the Goal to Learn Strengthens Learning in an Unintentional Learning Task James R. Schmidt \& Jan De Houwer Ghent University

Word count: 3,672

Author Note

This research was supported by Grant BOF09/01M00209 of Ghent University to Jan De Houwer. James R. Schmidt is a postdoctoral fellow of the Research Foundation - Flanders (FWO - Vlaanderen).

Correspondence concerning this article should be addressed to James R. Schmidt, Ghent University, Henri Dunantlaan 2, B-9000 Ghent, Belgium. E-mail: james.schmidt@ugent.be. 


\begin{abstract}
Previous research demonstrated that contingency learning can take place in the absence of the intention to learn. For instance, in the colour-word contingency learning task each distracting word is presented most often in a given target colour (e.g., "month" in red; "plate" in green) and less often in the other colours. Participants respond faster and more accurately when the word is presented in the expected relative to an unexpected colour even though there is no reason why they would have the intention to learn the contingencies between the words and the colours. It remains to be determined, however, whether learning in such situations would benefit or suffer from adding the goal to learn contingencies. In the reported experiment, half of the participants were informed that each word was presented most often in a certain colour and were instructed to try to learn these contingencies. The other half of the participants were not informed that contingencies would be present. Participants given the explicit learning goal produced a larger contingency effect in response times than the control participants. In contrast to some results from other learning paradigms, our results suggest that intentional learning adds to, rather than interferes with, unintentional learning, and we propose an explanation for some conflicting results.
\end{abstract}




\section{Goal-Dependent Learning in the Colour-Word Contingency Learning Paradigm: Adding the Goal to Learn Strengthens Learning in an Unintentional Learning Task}

Within the literature on human contingency learning (Schmidt, in press) several paradigms have emerged that have proved useful in the study of unintentional learning processes. One example of this is the colour-word contingency learning paradigm introduced by Schmidt and colleagues (Schmidt \& Besner, 2008; Schmidt, Crump, Cheesman, \& Besner, 2007; Schmidt \& De Houwer, 2011a, 2011b; Schmidt, De Houwer, \& Besner, 2010). In this paradigm, each distracting word is presented most often in a certain target colour (e.g., "month" most often in red, "plate" most often in green, etc.) and participants respond faster and more accurately to trials where the word is presented in its high contingency colour (e.g., "month" in red) relative to a low contingency colour (e.g., "month" in green). Contingency awareness is generally quite limited in this paradigm and learning seems to often occur without awareness (Schmidt et al., 2007). Because participants are typically unaware of the contingencies, it is unlikely that they have the intention to learn them. Moreover, instructions remain silent about the presence of the contingencies and participants are not asked to learn them. One could argue that participants might still form the intention to learn the contingencies in order to improve their performance in the reaction time task. However, the reaction time task is so simple (i.e., reacting to colours) and reaction times are so short that an intentional strategy to use contingency knowledge in order to improve performance is likely to backfire and actually hamper performance. Hence, the goal to learn does not appear to be necessary for colour-word contingency learning.

One issue that has received relatively less attention is whether colour-word contingency learning (and human contingency learning in general) is moderated by the goal to learn. In the current research, we investigated what impact explicit learning goals have on the amount of 
learning occurring in a paradigm that would otherwise result in unintentional learning, namely the colour-word contingency learning paradigm. We use the term "unintentional learning paradigm" to refer to a learning task that, in the absence of the instruction to learn contingencies, would result in unintentional learning.

Intuitively, one would imagine that the goal to learn can only have a positive effect on the resultant learning. However, previous work assessing the impact of goals on learning in unintentional learning paradigms revealed null results or even a counterintuitive decrease in the size of learning effects as the result of inducing the goal to learn. In artificial grammar learning work, Forkstam, Elwér, Ingvar, and Petersson (2008) compared learning in participants who were informed that there would be contingencies that made for an artificial grammar with learning in participants who were not informed. No effects of instruction on learning were found. Reber (1976), in contrast, actually observed decreased learning of the grammar when given the explicit goal to learn. These results suggest that the goal to learn interferes with unintentional learning.

Similar results have been reported in the evaluative conditioning literature. For instance, Fulcher and Hammerl (2001) paired some neutral haptic (touch) stimuli with positive haptic stimuli and others with other neutral haptic stimuli. A standard evaluative conditioning effect is observed when the positively-conditioned neutral stimuli are rated more positively than the neutrally-conditioned neutral stimuli. However, this pattern was reversed in the participants that were told to learn the contingencies. Hence, in this case, the explicit goal to learn seems to override the normal learning process rather than strengthen it.

In order to assess the role of goals on contingency learning in the colour-word contingency learning paradigm, half of the participants in our experiment were assigned to an 
instruction group and half to a control group. As is typical in most experiments on unintentional contingency learning, control participants were not told about the contingencies at the start of the experiment. In contrast, instructed participants were told that word-colour contingency relationships would be present in the task and that they should try to learn them. Subjective contingency awareness (i.e., verbal acknowledgement of noticing the contingencies) and objective contingency awareness (i.e., above chance guessing of which word went with which colour) were also assessed.

\section{Method}

\section{Participants}

Forty-six Ghent University Dutch speaking undergraduates participated in the experiment in exchange for $€ 4$.

\section{Apparatus}

Stimulus and response timing were controlled by E-Prime software (Experimental Software Tools, 2002). Using an AZERTY keyboard, participants pressed the "J" key for red, the "K" key for yellow, and the "L" key for green in the main learning task and the objective awareness task. Participants pressed the "j" key for "ja" (yes) or the "n" key for "nee” (no) on the subjective awareness question.

\section{Materials and Design}

Participants were seated approximately $60 \mathrm{~cm}$ from the screen. Stimuli were presented on a black background and consisted of three neutral five-letter Dutch words (onder [under], maand [month], plaat [plate]) presented in three different print colours (red, yellow, green). One word (e.g., onder) was presented most often (8 of 10 times) in red, another word (e.g., maand) most often in yellow, and the third (e.g., plaat) most often in green. Words were presented equally 
often ( 1 of 10 times) in each of the remaining two colours. High contingency trials are trials in which the word is presented in its most frequent colour. Low contingency trials are trials in which the word is presented in another colour. The contingency effect is the difference in response time or accuracy between these two types of trials. The high contingency colour for each word was randomly determined for each participant. Words were presented in bold, $18 \mathrm{pt}$. Courier New font. The RGB values for the display colours were 255,0,0 (red), 255,255,0 (yellow), and 0,255,0 (green).

\section{Procedure}

First, participants were told that they would be responding to the print colour of words. Only participants in the instructed group $(\mathrm{N}=23)$ were then given the following (translated) additional instructions:

Note: Each word in the experiment is presented most often in a certain color. Specifically, one word is presented most often in red, another word is presented most often in yellow, and a third word is presented most often in green. Try to learn which word is presented in which color as you perform the task.

Each trial began with a white fixation "+" for $150 \mathrm{~ms}$, followed by a blank screen for another $150 \mathrm{~ms}$, followed by a coloured word for $2000 \mathrm{~ms}$ or until a response was made. Following correct responses, the next trial started immediately. If the trial timed out or an incorrect response was made, "XXX" in white was presented for $500 \mathrm{~ms}$, followed by the next trial. We presented 200 trials, selected at random with replacement. Afterwards, contingency awareness was assessed. Participants were first asked whether they noticed the word-colour contingencies (subjective contingency awareness), with the question being essentially identical to the learning goal instruction. After this, participants were asked to guess which colour each word was presented most often in (objective awareness). 


\section{Results}

Mean correct response latencies and percentage error data were analyzed (see Figure 1). Trials on which participants failed to respond (less than $1 \%$ of the data) were removed from analyses. The correlations used for the awareness data are nonparametric Spearman's $\rho$, which better controls for the influence of outliers with small sample sizes. Correlations with parametric Pearson's $r$ were essentially identical.

\section{Response Latencies}

An ANOVA for response latencies with the factors of contingency (high vs. low) and group (instructed vs. control) revealed a significant main effect of contingency, $F(1,44)=$ 46.737, $M S E=1506, p<.001, \eta_{p}^{2}=.51$, indicating overall faster responses for high relative to low contingency trials. The main effect of group was not significant, $F(1,44)=1.918, M S E=$ $11569, p=.173, \eta_{p}^{2}=.04$. Critically, the interaction between contingency and group was significant, $F(1,44)=4.419, M S E=1506, p=.041, \eta_{p}^{2}=.09$, indicating a significantly larger contingency effect for the instructed group relative to controls. Planned comparisons revealed that the contingency effect was significant both for participants in the instructed group (high: 547 ms; low: $620 \mathrm{~ms}), t(22)=5.583, S E_{\text {diff }}=13, p<.001, \eta_{p}^{2}=.59$, and for participants in the control group (high: $533 \mathrm{~ms}$; low: $572 \mathrm{~ms}$ ), $t(22)=3.950, S E_{\text {diff }}=10, p<.001, \eta_{p}^{2}=.38$.

\section{(Figure 1 about here)}

\section{Percentage Error}

An ANOVA for errors with the factors of contingency (high vs. low) and group (instructed vs. control) revealed a significant main effect of contingency, $F(1,44)=24.604, M S E$ $=16, p<.001, \eta_{p}^{2}=.35$, indicating overall less errors on high relative to low contingency trials. 
The main effect of group was also significant, $F(1,44)=5.177, M S E=28, p=.028, \eta_{p}^{2}=.10$, showing fewer errors in the control group than in the instructed group. Although numerically in the expected direction, the interaction between contingency and group was not significant, $F(1,44)=.943, M S E=16, p=.337, \eta_{p}^{2}=.02$. Planned comparisons revealed that the contingency effect was significant both for participants in the instructed group (high: 5.0\%; low: $9.9 \%), t(22)=3.999, S E_{\text {diff }}=1.2, p<.001, \eta_{p}^{2}=.42$, and for participants in the control group (high: $3.3 \%$; low: $6.6 \%$ ), $t(22)=2.973, S E_{\text {diff }}=1.1, p=.007, \eta_{p}^{2}=.29$.

\section{Contingency Awareness}

Overall, contingency awareness was high in this experiment, probably due to the very strong contingency manipulation (i.e., $80 \%$ high contingency trials). In the instructed group, 21 of 23 participants (91\%) said that they were aware of the word-colour contingencies (subjective awareness). In the control group, 17 of 23 participants (74\%) said that they were aware. This four participant $(17 \%)$ difference is suggestive, but was not statistically significant, $t(44)=$ $1.563, S E_{\text {diff }}=11, p=.125, \eta_{p}^{2}=.05$. Subjective awareness did, however, correlate significantly with the size of the error contingency effect, $\rho(44)=.349, p=.017$, and marginally with the response time contingency effect, $\rho(44)=.268, p=.072$. Objective awareness was $96 \%$ in the instructed group and $88 \%$ in the control group. This $8 \%$ difference was again suggestive, but not significant, $t(44)=1.003, S E_{d i f f}=7, p=.321, \eta_{p}^{2}=.02$. These rates of objective awareness were well above chance (33\%) in both the instructed, $t(22)=19.547, S E=3, p<.001, \eta_{p}^{2}=.95$, and control conditions, $t(22)=8.468, S E=7, p<.001, \eta_{p}^{2}=.77$. Like the subjective awareness measure, objective awareness was significantly related to the size of the error contingency effect, $\rho(44)=.311, p=.035$, and marginally with the response time contingency effect, $\rho(44)=.259, p$ 
$=.083$.

\section{Discussion}

In the reported study, we assessed the impact of explicit learning goals on the size of the colour-word contingency learning effect. Our results demonstrated a clear beneficial effect. Relative to participants in the control condition, participants given contingency learning instructions showed an $87 \%$ significant increase in the size of the response time effect and a $48 \%$ nonsignificant numerical increase in the size of the error effect. Thus, our data show that the conscious intent to learn can have a beneficial effect on the resultant unintentional learning.

On the other hand, a small number of earlier studies concerning the effects of goals on artificial grammar learning (e.g., Reber, 1967) and evaluative conditioning (e.g., Fulcher \& Hammerl, 2001) revealed a negative effect of the goal to learn on contingency learning. One interpretation of these negative effects is that the goal to learn activates explicit learning processes that counteract and sometimes overrule implicit learning processes (e.g., Reber, 1989; see Lieberman, Chang, Chiao, Bookheimer, \& Knowlton, 2004, for neurological evidence). Such an account, of course, has difficultly explaining the beneficial effects of goals in the present experiment.

One could posit, however, that the negative effects of the goal to learn in artificial grammar learning studies is related to the complexity of the information to be learned in those studies (i.e., the artificial grammar). This would imply that the goal to learn is beneficial when the to-be-learned information is simple (e.g., the simple contingencies in our study) but detrimental when the to-be-learned information is complex. If participants are left to rely on unintentional learning only, then they might be more effective at picking up the more complex relationships, whereas the introduction of a learning goal causes participants to rely on simpler 
(but less correct) contingencies. This account in terms of difficulty does not, however, explain the negative effects of the goal to learn in evaluative conditioning studies. As in our study, evaluative conditioning studies typically involve only simple contingencies between two stimuli.

Another possibility is that the difference results from the use of judgment versus performance tasks. Some have argued that judgement tasks (i.e., tasks in which learning is assessed on the basis of judgements; e.g., a standard evaluative conditioning task) and performance tasks (i.e., tasks in which learning is inferred from reaction time performance; e.g., colour-word contingency) are driven by inherently different types of learning (see Shanks \& St. John, 1994, for a review). It may be the case that the harmful effects of intentional learning in evaluative conditioning and artificial grammar learning are due to the use of a classification judgement response (i.e., judgements of liking or grammaticality). For instance, in an evaluative taste conditioning experiment participants may normally learn which neutral taste goes with which valenced taste and subsequently rate neutral stimuli based on the valenced stimuli with which they were paired. However, when given explicit learning instructions participants may feel that their subsequent ratings of the neutral stimuli should not be based on the pairings and overcompensate their valence ratings in the reverse direction. Thus, the learning of the relationships between stimuli may not actually be impaired. Indeed, contingency awareness is generally increased under such conditions (Baeyens, Eelen, \& Van den Bergh, 1990). Rather, participants might be merely changing how they decide to classify stimuli when given different instructions. Because participants can easily control their judgements, learning as assessed by judgements is very susceptible to these kinds of conscious strategies.

A similar argument could be made for the reduced learning effects of Reber (1967) with explicit learning instructions. Because participants in the instructed group were told to explicitly 
learn the grammar they might be less inclined to base their judgements on intuition or gut feelings. Thus, unintentional learning (which might be more potent than intentional learning) would be undermined. As with the evaluative conditioning work participants may not be learning contingencies to a lesser extent. Their intuitions may still be shaped by the contingencies. Rather, they may simply be expressing that learning differently with a change in their approach to categorization after being giving learning instructions. In other words, their conscious strategy for making judgements might lead to the absence or reversal of the effect. In that sense, instructions in such paradigms reduce the observed learning effect, but not learning per se.

For this reason, a performance (i.e., response time) task such as the colour-word contingency learning task will not produce this same detrimental effect of explicit learning instructions. As noted earlier, it is unlikely that participants intentionally use the contingencies to speed colour identification performance, particularly given the speeded nature of the task. In fact, participants probably have little control over how learned information about contingencies influences their reaction time performance, thus making it unlikely that a conscious strategy to use or not use contingency knowledge will counteract unintentional learning. More generally, the explicit instruction to learn contingencies might always strengthen learning, perhaps even in the tasks that have previously shown negative effects (e.g., where the benefit is concealed by the costs associated with a categorization response). More work, of course, is warranted before making any strong conclusions on this matter. Whatever the actual cause of the discrepancy between our findings and previous findings, our study clearly shows that the goal to learn contingencies can facilitate learning, even in a paradigm that would otherwise result in unintentional learning. 
Consistent with our findings, some other recent research has shown positive effects of goals on learning, though with goals of a different sort. In two unconscious goal pursuit experiments, Eitam, Hassin, and Schul (2008) demonstrated that implicitly-primed performance goals (i.e., the goal to perform well in the task) had a facilitative effect on implicit learning effects. Similarly, Corneille, Yzerbyt, Pleyers, and Mussweiler (2009) primed participants with the implicit goal to process similarities versus differences between stimuli and found that the goal to process similarities between stimuli lead to larger evaluative conditioning effects. Although performance goals such as these are clearly quite different than the goal to learn used in the present investigation, these past reports are consistent with our findings in showing that goals can have a positive influence on the amount of learning observed.

The question then arises as to what mechanism leads to an increase in learning with the introduction of a learning goal. One possibility is that goals lead to explicit knowledge about the contingency relations and that this explicit knowledge is used in combination with the unintentionally-learned contingencies to produce a larger contingency effect. Indeed, while contingency learning in our paradigm seems to often occur without awareness (for related work, see Carlson \& Flowers, 1996; Destrebecqz \& Cleeremans, 2001; Jiménez \& Méndez, 1999; Lewicki, Hill, \& Czyzewska, 1992; Mayr, 1996; McKelvie, 1987; Nissen \& Bullemer, 1987; Song, Howard, \& Howard, 2007), the present results suggest that contingency awareness might help, as indicated by the positive correlations between awareness and the learning effects.

Another possibility is that explicit goals lead to an increase in attention to the predictive dimension (i.e., the word in our paradigm) as a result of the fact that the predictive dimension is more relevant to the task. Indeed, work with various paradigms has demonstrated that attention to the predictive dimension is crucial for contingency learning to occur (e.g., Eitam, Schul, \& 
Hassin, 2009; Jiang \& Chun, 2001; Jiménez \& Méndez, 1999). Thus, it could be the case that participants in the instructed group attend more to the word than participants in the control condition, which could lead to stronger encoding of the current trial and/or retrieval of previously encountered trials. Whatever the mechanism that mediates the impact of goals to learn on unintentional learning, our results show that the goal to learn can improve unintentional learning. As such, it sheds new light on the relation between goals and learning in general. 


\section{References}

Baeyens, F., Eelen, P., \& Van den Bergh, O. (1990). Contingency awareness in evaluative conditioning: The case for unaware effective-evaluative learning. Cognition \& Emotion, 4, 3-18.

Corneille, O., Yzerbyt, V. Y., Pleyers, G., \& Mussweiler, T. (2009). Beyond awareness and resources: Evaluative conditioning may be sensitive to processing goals. Journal of Experimental Social Psychology, 45, 279-282.

Carlson, K. A., \& Flowers, J. H. (1996). Intentional versus unintentional use of contingencies between perceptual events. Perception \& Psychophysics, 58, 460-470.

Destrebecqz, A., \& Cleeremans, A. (2001). Can sequence learning be implicit? New evidence with the process dissociation procedure. Psychonomic Bulletin \& Review, 8, 343-350.

Experimental Software Tools. (2002). E-Prime. Website <http://www.pst-net.com>.

Eitam, B., Hassin, R. R., \& Schul, Y. (2008). Nonconscious goal pursuit in novel environments. Psychological Science, 19, 261-267.

Eitam, B., Schul, Y., \& Hassin, R. R. (2008). Goal relevance and artificial grammar learning. Quarterly Journal of Experimental Psychology, 62, 228-238.

Forkstam, C., Elwér, Å., Ingvar, M., \& Petersson, K. (2008). Instruction effects in implicit artificial grammar learning: A preference for grammaticality. Brain Research, 1221, 8092.

Fulcher, E. O., \& Hammerl, M. (2001). When all is revealed: A dissociation between evaluative learning and contingency awareness. Consciousness and Cognition, 10, 534-549.

Jiang, Y. H., \& Chun, M. M. (2001). Selective attention modulates implicit learning. Quarterly Journal of Experimental Psychology, 54A, 1105-1124. 
Jiménez, L., \& Méndez, C. (1999). Which attention is needed for implicit sequence learning? Journal of Experimental Psychology: Learning, Memory, and Cognition, 25, 236-259.

Lewicki, P., Hill, T., \& Czyzewska, M. (1992). Nonconscious acquisition of information. American Psychologist, 47, 796-801.

Lieberman, M. D., Chang, G. Y., Chiao, J., Bookheimer, S. Y., \& Knowlton, B. J. (2004). An event-related fMRI study of artificial grammar learning in a balanced chunk strength design. Journal of Cognitive Neuroscience, 16, 427-438.

Mayr, U. (1996). Spatial attention and implicit sequence learning: Evidence for independent learning of spatial and nonspatial sequences. Journal of Experimental Psychology: Learning, Memory, and Cognition, 22, 350-364.

McKelvie, S. J. (1987). Learning and awareness in the Hebb digits task. Journal of General Psychology, 114, 75-88.

Nissen, M. J., \& Bullemer, P. (1987). Attentional requirements of learning: Evidence from performance measures. Cognitive Psychology, 19, 1-32.

Reber, A. S. (1976). Implicit learning of synthetic languages: Role of instructional set. Journal of Experimental Psychology: Human Learning and Memory, 2, 88-94.

Reber, A. S. (1989). Implicit learning and tacit knowledge. Journal of Experimental Psychology: General, 118, 219-235.

Schmidt, J. R. (2012). Human contingency learning. In N. M. Seal (Ed.), Encyclopedia of the sciences of learning (pp. 1455-1456). New York: Springer.

Schmidt, J. R., \& Besner, D. (2008). The Stroop effect: Why proportion congruent has nothing to do with congruency and everything to do with contingency. Journal of Experimental Psychology: Learning, Memory, \& Cognition, 34, 514-523. 
Schmidt, J. R., Crump, M. J. C., Cheesman, J., \& Besner, D. (2007). Contingency learning without awareness: Evidence for implicit control. Consciousness and Cognition, 16, 421435.

Schmidt, J. R., \& De Houwer, J. (2011a). Does temporal contiguity moderate contingency learning in a speeded performance task? Manuscript submitted for review.

Schmidt, J. R., \& De Houwer, J. (2011b). Contingency learning with evaluative stimuli: A performance paradigm. Manuscript submitted for review.

Schmidt, J. R., De Houwer, J., \& Besner, D. (2010). Contingency learning and unlearning in the blink of an eye: A resource dependent process. Consciousness and Cognition, 19, 235250.

Shanks, D. R., \& St. John, M. F. (1994). Characteristics of dissociable human learning systems. Behavioral and Brain Sciences, 17, 367-395.

Song, S., Howard, J. H., Jr., \& Howard, D. V. (2007). Implicit probabilistic sequence learning is independent of explicit awareness. Learning \& Memory, 14, 167-176. 
Figures

Figure 1. Response latencies, standard errors, and error percentages as a function of contingency and instruction group. 


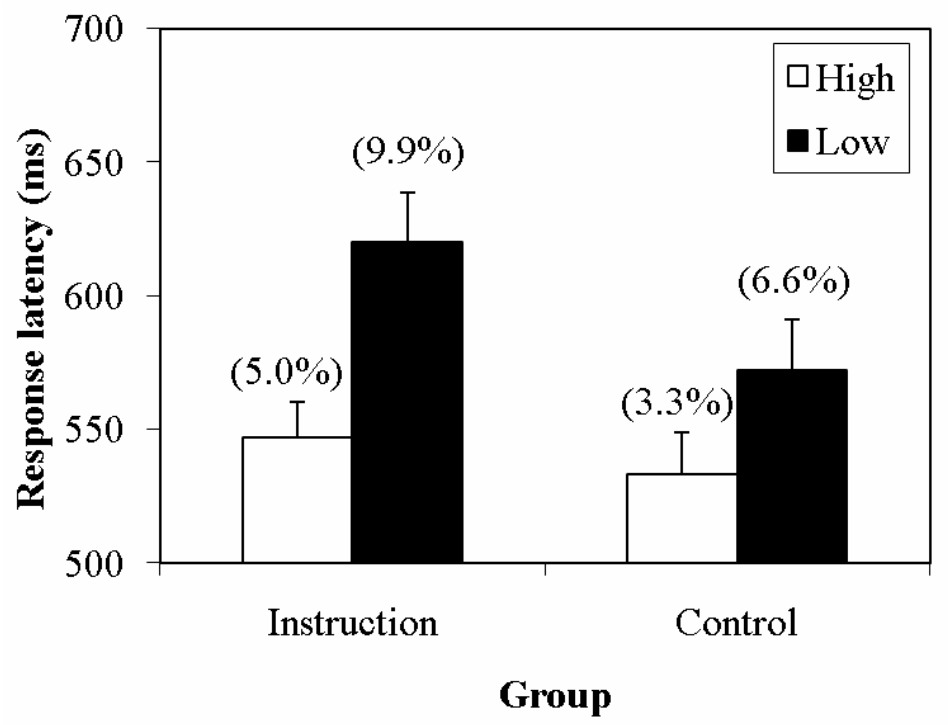

